\title{
Behavior of condensed droplets growth and jumping on superhydrophobic surface
}

\author{
Sihang $\mathrm{Gao}^{1}$, Fuqiang $\mathrm{Chu}^{2}$, Xuan Zhang ${ }^{1}$ and Xiaomin $\mathrm{Wu}^{1, *}$ \\ ${ }^{1}$ Key Laboratory for Thermal Science and Power Engineering of the Ministry of Education, \\ Department of Energy and Power Engineering, Tsinghua University, Beijing 100084, China \\ ${ }^{2}$ School of Aeronautic Science and Engineering, Beihang University, Beijing 100191, China
}

\begin{abstract}
Droplets on the superhydrophobic surface can fall off the surface spontaneously, which greatly promote dropwise condensation. This study considers a continuous droplet condensation process including droplet growth and droplet jumping. A droplet growth model considered NCG is developed and droplet jumping is simulated using VOF (Volume Of Fluid) model. Al-based superhydrophobic surfaces are prepared using chemical deposition and etching method. The Al-based superhydrophobic surface has a contact angle of $157^{\circ} \pm 1^{\circ}$ and a rolling angle of $2^{\circ} \pm 1^{\circ}$. An observation experiment is designed to observe droplet jumping on superhydrophobic surface using a high- speed camera system. The result of droplet growth model shows a good match with experimental data in mid-term of droplet growth. For droplet jumping, simulation and experiment results show that droplet jumping of different diameter has a universality in a non-dimensional form. The jumping process can be divided into 3 stages and droplet vibration is observed.
\end{abstract}

\section{INTRODUCTION}

Compared with filmwise condensation, dropwise condensation has a higher heat transfer coefficient, which can be applied for heat transfer enhancement ${ }^{[1,2]}$. The key point of maintaining dropwise condensation is the quick removal of the condensate from the surface. Many studies have proven that droplets on a superhydrophobic surface with high contact angle and rolling angle can fall off the surface spontaneously, which greatly promote dropwise condensation ${ }^{[1-6]}$. Therefore, it is of great importance to study condensed droplet growth and jumping behavior on superhydrophobic surface.

Many studies have worked on the mechanism of droplet growth both theoretically and experimentally. In 1960 s, Le and Rose et a ${ }^{[3]}$ built a single droplet growth model based on heat conduction theory. Abu Orabi ${ }^{[7]}$ made an improvement to the growth mode by applying thermal resistance theory. Tanaka ${ }^{[8]}$ used group balanced theory on multiple droplets growth model and gave two types of multiple droplets growth model: direct vapor accumulation and droplet coalesce. Considering the influence of surface wettability, Kim et al ${ }^{[4]}$ expanded droplet thermal model to surface with contact angle larger than $90^{\circ}$ and gave the calculation of curvature thermal resistance in droplet growth model based on heat transfer theory. Miljkovic et al ${ }^{[9]}$ found that the thermal contact resistance between Cassie-state droplet and substrate has a significant influence on droplet growth.
Rykaczewski et al ${ }^{[10]}$. compared two growth models: CB (constant base) model and CCA (constant contact angle) model on the superhydrophobic surface with an ESEM observation for droplets growth. They found that droplet diameter increases in a step like fashion of $\mathrm{CB}$ and CCA mode.

Massive attention has been paid to droplet jumping behavior on the superhydrophobic surface due to the great potential for heat transfer enhancement. Chen et $a l^{[1]}$. First reported droplet coalescence and jumping behavior on a superhydrophobic surface. The selfpropelled jumping phenomenon is triggered by coalescence between adjacent droplets and the released surface energy is transformed into kinetic energy. Further study showed that droplet jumping velocity of droplet smaller than capillary size can reach $1 \mathrm{~m} / \mathrm{s}^{[2]}$. Lv et $a l^{[11]}$ found that viscosity dissipation has a significant influence on surface energy. Enright et al. ${ }^{[12]}$ observed droplet coalescence in sub-10 $\mu \mathrm{m}$ size. Results showed that the velocity of the coalescent droplet is up to $1.4 \mathrm{~m} / \mathrm{s}$ with a maximum surface energy conversion rate of $6 \%$.

Despite significant achievement in droplet growth modeling and droplet jumping, a systematic study on droplet condensation on the superhydrophobic surface is still lacking. For droplet growth, molecule transfer in the nucleation stage needs to be taken into consideration. For droplet coalescence and jumping, the characterization of the jumping process need to be

\footnotetext{
* Corresponding author: wuxiaomin@mail.tsinghua.edu.cn
} 
specified and influence factor on surface energy conversion needs more attention.

This work focuses on the mechanism of droplet growth and droplet jumping on the superhydrophobic surface. A droplet growth model considered NCG is developed while droplets jumping are simulated using VOF model. An observation experiment is designed to observe droplets jumping with different volume on the superhydrophobic surface using a high-speed camera. Moreover, a chemical deposition and etching method ${ }^{[13]}$ is applied to make Al-based superhydrophobic surface. The result of droplet growth model shows a good match with experimental data in mid-term of droplet growth while in the early stage and late stage the model has significant differences from experiment data because of the coalesce between microdroplets. As for droplets jumping, results show that there is a universality of velocity change in the process of droplets jumping. Surface energy conversion rate of droplet varies little with different volume. In the observation experiment, 3 stages for droplets jumping are confirmed, and droplet shaking is observed in the third stage. In the process of droplet shaking, the length of the coalesced droplet decreases quickly while the height of the coalesced droplet increases. The result of observation experiment matches well with simulation.

\subsection{Droplet growth model}

The droplet diameter experiences a wide range of change during droplet growth, which indicates that the heat and mass transfer mechanism is different in different diameter. $K n$ number is used to characterize the dominant growth mechanism ${ }^{[14]}$. The critical value for $K n$ number is set as 0.1 . When $K n$ number is larger than 0.1 , the droplet growth is modeled using a molecular dynamic theory ${ }^{[15]}$. In this stage, droplet grows by the coalescence of water molecules in the moist air. When $K n$ number is less than 0.1 , droplet growth can be modeled by a thermal resistance theory. The radius growth rate is given as:

$$
\begin{gathered}
\frac{\mathrm{d} r}{\mathrm{~d} t}=\frac{D_{v} M_{v}\left(p_{\text {sat }}-p_{\text {sat }}^{*}\right)}{\rho_{l} N_{a} \sqrt{2 \pi m_{v} k T}}, K n \geq 0.1 \\
\frac{\mathrm{d} r}{\mathrm{~d} t}=\frac{\Delta T\left(1-\frac{r_{\min }}{r}\right)}{2 H_{l} \rho_{l}\left(\frac{1}{h}+\frac{r \theta(1-\cos \theta)}{4 k \sin \theta}+\frac{\delta_{w}(1-\cos \theta)}{k_{w} \sin ^{2} \theta}+\frac{\delta}{\pi k_{e} r^{2} \sin ^{2} \theta}\right)} \\
, K n<0.1
\end{gathered}
$$

\subsection{Droplet jumping simulation}

Self-propelled droplet jumping process is simulated using a VOF model. 4 groups of droplets with a diameter varying from $600 \mu \mathrm{m}$ to $1200 \mu \mathrm{m}$ are set to characterize the jumping process and surface energy conversion. In our case, two droplets are symmetrically placed in the center of the plane. Ambient temperature is $20^{\circ} \mathrm{C}$ with a relative humidity of 0.7 . Water and air property is selected according to the ambient temperature. For energy analysis, the surface energy and kinetic energy are given by

$$
\begin{gathered}
E_{\text {surface }}=\sigma_{\mathrm{lg}} S_{\mathrm{lg}} \\
E_{k}=m u^{2} / 2
\end{gathered}
$$

The computational domain is a $4 \times 4 \times 2 \mathrm{~mm}$ cuboid with two droplets placed in the center, as shown in Fig.1. Boundary condition in the bottom surface is a non-slip wall condition ${ }^{[16]}$ with a static contact angle of $157^{\circ}$. The top surface is set as an open condition and wall condition is applied to the vertical surface.

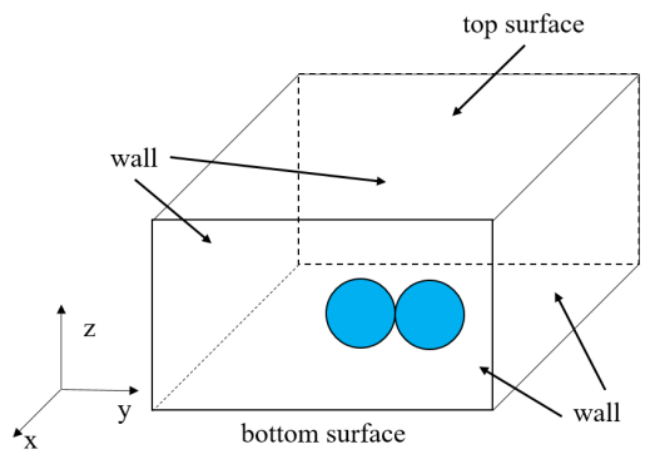

Fig. 1. Schematic of computational domain.

\subsection{Observation experiment}

The computational domain is a $4 \times 4 \times 2 \mathrm{~mm}$ cuboid with two droplets placed in the center, as shown in Fig.1. Boundary condition in the bottom surface is a non-slip wall condition $^{[16]}$ with a static contact angle of $160.2^{\circ}$. The top surface is set as an open condition and wall condition is applied to the vertical surface.

For better understanding of droplet jumping process, an experiment is designed to observe droplet jumping on a prepared superhydrophobic surface. The result of observation experiment is compared with simulation result. Droplet jumping process is imaged using a highspeed camera, as shown in Fig.2. Two droplets are placed on the Al-based superhydrophobic substrate by a $5 \mu \mathrm{L}$ micro-injector. Droplets coalescence is triggered by air blow and the following jumping process is recorded by the high-speed camera. The Al-based superhydrophobic surface is fabricated using a chemical etching and deposition method ${ }^{[13]}$. The static contact angle of the prepared surface is $160.2^{\circ} \pm 1.5^{\circ}$ and the rolling angle is $2^{\circ} \pm 1^{\circ}$, measured by a JC2000C 1 measuring instrument in ambient temperature.

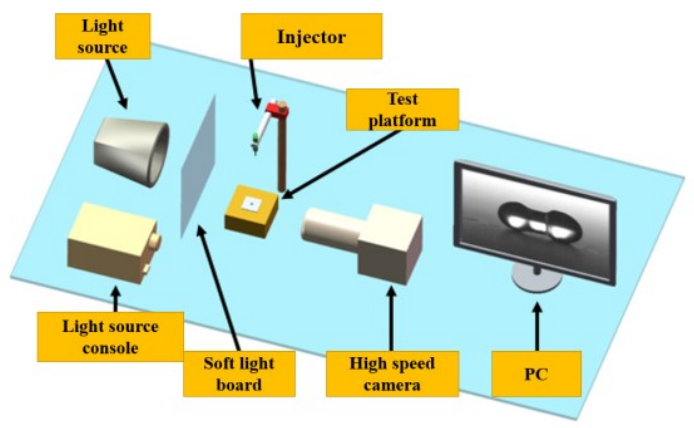

Fig. 2. Schematic diagram of the experimental system. 


\section{RESULTS AND DISCUSSION}

\subsection{Droplet growth model}

The droplet growth model is compared with experiment data from previous study ${ }^{[17]}$. The parameters of the growth model are consistent with experiment. As shown in Fig.3, the growth model matches well with experiment data in middle stage (420-550s). However, there is a significant discrepancy in early stage $(0-420 \mathrm{~s})$ and late stage (550-800s). In experiment, there is a nucleation time before droplets begin to grow, which leads to a time delay compared with the growth model. In the late stage, droplet is big enough so that droplet coalescence happens between microdroplets, causing a faster droplet growth in the experimental situation.

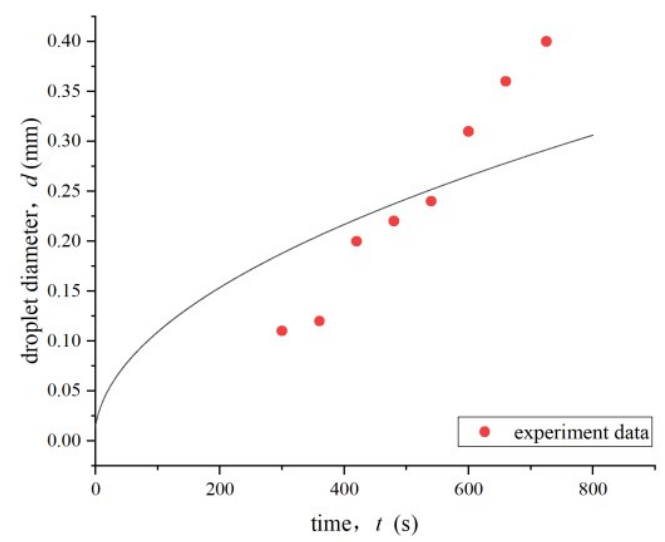

Fig. 3. Droplet diameter vs time.

\subsection{Droplet shape transformation and shaking phenomenon during jumping process}

Results of the simulation are compared with images captured in the observation experiment. Fig.4 is the droplet shape from experiment observation and simulation in different time node. A dimensionless method is applied to help judge droplet shape transformation.

Droplet shape evolution process: result from observation experiment shows that coalescence happens with formation of liquid bridge. During the coalescence process $(0-3 \mathrm{~ms})$, the interspace between the droplets is filled with the growing of liquid bridge. Original small droplet gradually disappears and the liquid bridge changes into coalescent droplet. The liquid bridge keeps growing and touches the substrate while the ends of the droplets shrink. After the coalescence, the new droplet jump off the substrate and its velocity decrease continuously under the influence of gravity. Compared with the simulation result in dimensionless form, droplet shape in same time node matches well though there is a difference in droplet volume. The effect of droplet volume will be discussed in the following part.

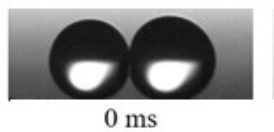

0

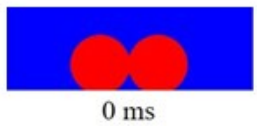

$\mathrm{ms}$

0

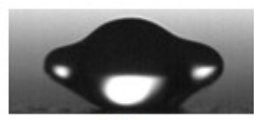

$1.5 \mathrm{~ms}$

0.40

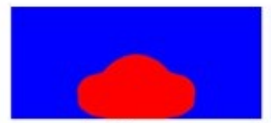

$0.69 \mathrm{~ms}$

0.40

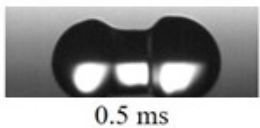

0.13

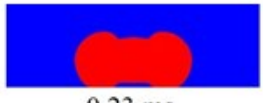

$0.23 \mathrm{~ms}$

0.13

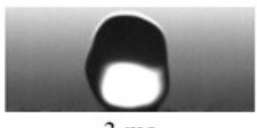

$3 \mathrm{~ms}$

0.81

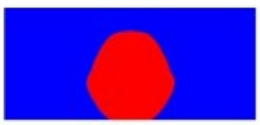

$1.39 \mathrm{~ms}$

0.81

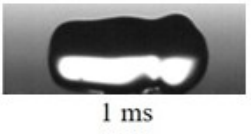

0.27
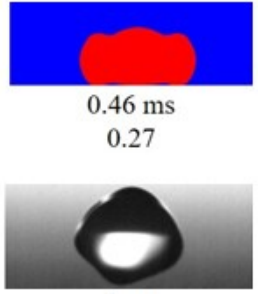

$4 \mathrm{~ms}$

1.07

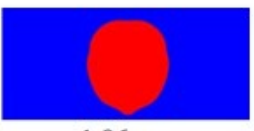

$1.86 \mathrm{~ms}$

1.07
Fig. 4. Droplet shape evolution during jumping process

Droplet shaking phenomenon: after the coalescent droplet takes off from the substrate, a droplet shaking phenomenon is found both in observation experiment and simulation cases. Fig.5 shows the ratio of droplet width and height with dimensionless time. There is a quick increase in droplet height during coalescence while the width decreases. After the leave from substrate, both the height and the width change without an obvious rule. The cause of droplet shaking is the asymmetry of the coalescence, as shown in Fig.1. The shrinking of the coalescent droplet in y direction causes the increasing in $\mathrm{x}$ and $\mathrm{z}$ direction. However, droplet shape in $z$ direction is unavailable due to the limitation of equipment. The effect of viscosity and gravity need to be further studied.

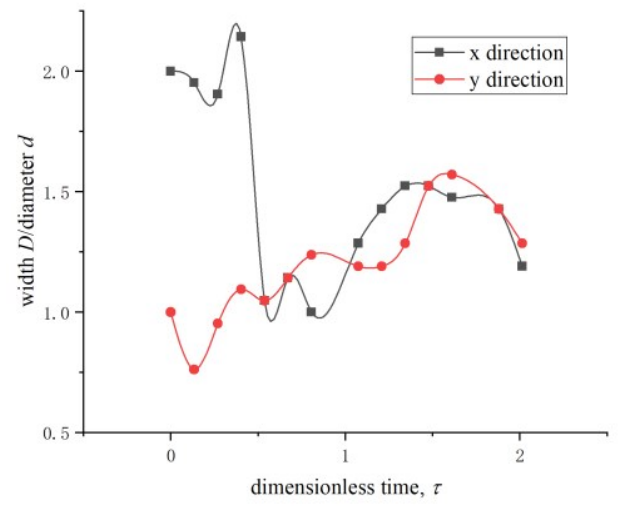

Fig. 5. Ratio of droplet height and width.

\subsection{Characterization of droplet jumping process}

The jumping velocity obtained from simulation is shown in Fig.6, from which three stages of droplet jumping process can be divided ${ }^{[5]}$ : (a) liquid bridge extension. The liquid bridge forms between two droplets and extends to the substrate. The velocity in this stage changes a little because the liquid bridge has not touched the substrate. (b) Rebounding. After the liquid bridge touched the substrate, the coalescent droplet begins to 
form. The velocity of the droplet shows a rapid increase with surface energy released and transferred into kinetic energy. However, there is a turning point at the end of this stage. This is the effect of gravity and viscosity when the droplet is about to jump from the substrate. (c) Free motion. In this stage, the droplet is completely separated from the substrate and the velocity continuously decreases under the influence of gravity and viscosity.

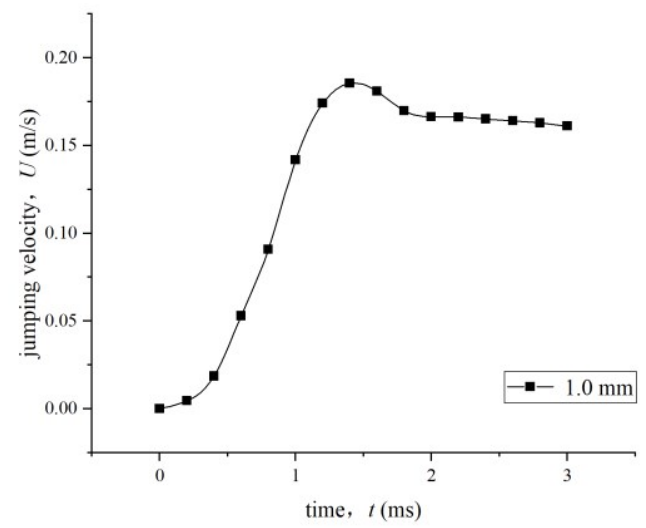

Fig. 6. Ratio of droplet height and width.

Volume effect on droplet velocity: jumping velocity of different droplet volume is plotted in Fig.7 (a). The maximum velocity decreases as the droplet volume increases. And smaller droplet gets to the maximum velocity faster than larger droplets, which suggests a faster surface energy conversion rate. Considering the surface energy and kinetic energy equation, the relationship between jumping velocity $U$ and droplet diameter can be given as:

$$
U \sim \sqrt{\frac{\sigma_{\mathrm{lg}}}{d}}
$$

This also suggests that jumping velocity decreases with the increase of droplet diameter, which corresponds to the simulation result. A dimensionless analysis is applied to the velocity-time scheme based on Boreyko and Chen's study ${ }^{[1,2]}$. The dimensionless velocity $v$ and time $\tau$ are calculated as:

$$
\begin{gathered}
v=\sqrt{\sigma / \rho r} \\
\tau=\sqrt{\rho r^{3} / \sigma}
\end{gathered}
$$

As shown in Fig.7 (b), the dimensionless curves from different volume show the similarity, which suggests that there is a uniformity in droplet jumping behavior in simulation cases. This finding can help predict droplets jumping velocity in two symmetric droplets coalescent cases.

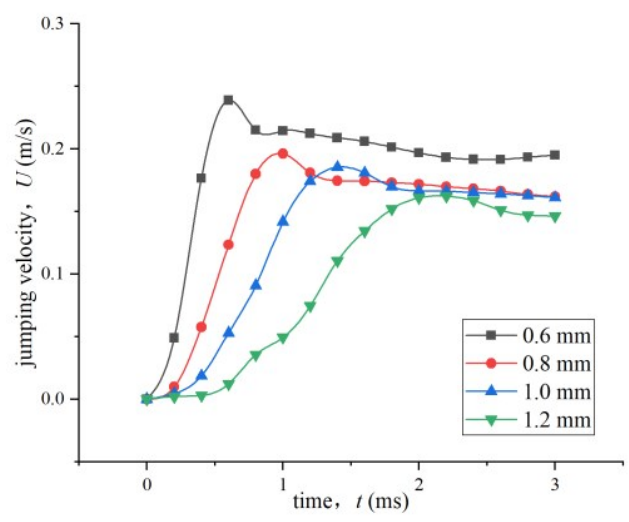

(a)

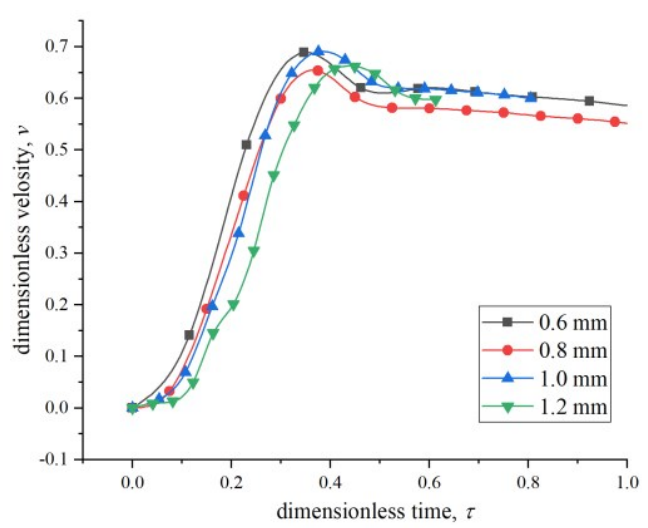

(b)

Fig. 7. Velocity vs time curve: (a) Normal case; (b) Dimensionless case.

Energy conversion: the energy conversion and the jumping velocity are plotted in Fig.8. Fig.8 (a) is variation trend of surface energy and kinetic energy. Compared with surface energy, the kinetic energy is much smaller. To further study the conversion of these two energy, the ratio of kinetic energy and surface energy is calculated and plotted in Fig.8 (b). The energy conversion ratio of the 4 cases in this work is around $5.5 \%$, which is independent of droplet volume. It is demonstrated that a constant percentage of surface energy is converted to kinetic energy, independent of droplet volume.

In previous part the relationship between droplet diameter and jumping velocity is given in equation (5). The maximum jumping velocity is shown in Fig.8(c) and a polynomial fitting is applied to the data. The fitting function is given as:

$$
U=\frac{0.20452}{\sqrt{d}}-0.02967, R=0.977
$$

The fitting result matches well with the velocity relationship, suggesting a credible prediction for droplet jumping velocity. 


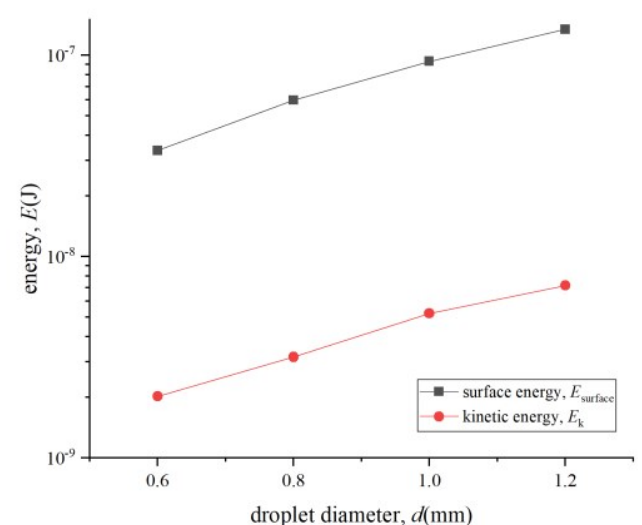

(a)

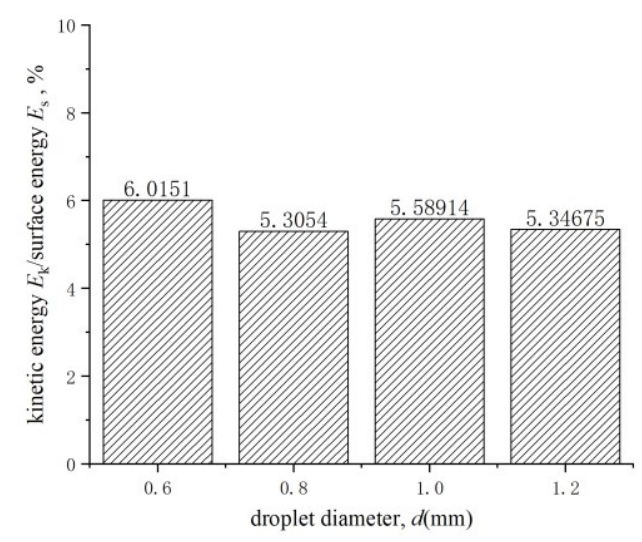

(b)

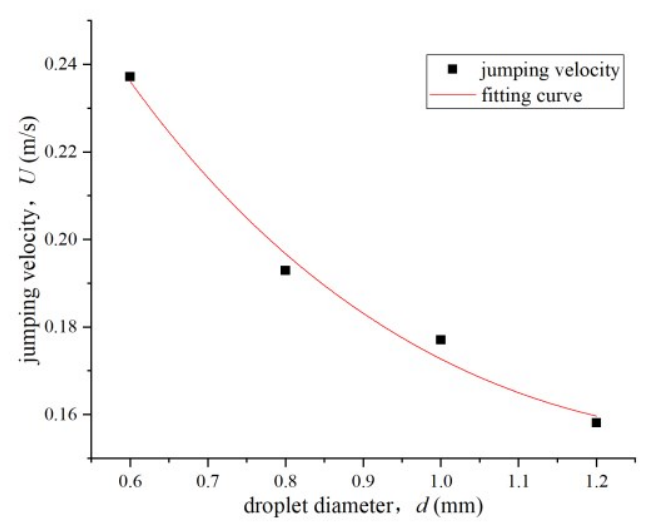

(c)

Fig. 8. Energy conversion of different volume: (a) Surface energy and kinetic energy; (b) Energy conversion ratio; (c) Maximum jumping velocity.

\section{CONCLUSIONS}

In this work, a theoretical model was developed to reveal the effect factors for droplet growth on the superhydrophobic surface. The growth of microdroplets is simulated firstly by a molecular dynamics based model and then a thermal resistance model. The newlybuilt model matches well with experiment data in middle stage but has a significant difference in early and later stage, which can be explained by the nucleation time and microdroplets coalescence in the experimental situation. Next, droplet jumping phenomenon is simulated using a VOF model to characterize the droplet shape evolution process and effect factors on energy conversion. Lastly, an observation experiment was conducted to verify the results of the simulation. The result of simulation matches well with experiment and droplet shaking was observed in the jumping process. It was found that the energy conversion ratio is around $5.5 \%$, independent of droplet diameter, which causes a decrease in jumping velocity with the increase in droplet diameter. These results can help describe the droplet jumping process which is crucial to modeling the dropwise condensation on superhydrophobic surface.

\section{Acknowledgements}

This work was supported by the National Natural Science Foundation of China (No. 51976098).

\section{References}

1. Chen, C.-H., Cai, Q., Tsai, C., Chen, C.-L., Xiong, G., Yu, Y., and Ren, Z., Applied Physics Letters, 90(17), p. 173108 (2007).

2. Boreyko, J. B., and Chen, C.-H., Physical Review Letters, 103(18), p. 184501 (2009).

3. Fevre, E. J. L., and Rose, J. W., A theory of heat transfer by dropwise condensation, 1966.

4. Kim, S., and Kim, K. J., Journal of Heat Transfer, 133(8), p. 081502 (2011).

5. Liu, T. Q., Sun, W., Sun, X. Y., and Ai, H. R., Colloids and Surfaces A: Physicochemical and Engineering Aspects, 414, pp. 366-374 (2012).

6. Chu, F., Wu, X.M., and Ma, Q., 2017, Applied Thermal Engineering, 115, pp. 1101-1108 (2017).

7. Abu-Orabi, M., 1998, International Journal of Heat \& Mass Transfer, 41(1), pp. 81-87 (1998).

8. Tanaka, H., International Journal of Heat \& Mass Transfer, 27(27), pp. 327-335 (1975).

9. Miljkovic, N., Enright, R., and Wang, E. N., Journal of Heat Transfer, 135(11), pp. 111004-111004111014 (2013).

10. Rykaczewski, K., Langmuir, 28(20), pp. 7720-7729 (2012).

11. Lv, C., Hao, P., Yao, Z., Song, Y., Zhang, X., and He, F., Applied Physics Letters, 103(2), p. 1103 (2013).

12. Enright, R., Miljkovic, N., Sprittles, J., Nolan, K., Mitchell, R., and Wang, E. N., Acs Nano, 8(10), pp. 10352-10362 (2015).

13. Chu, F., and Wu, X.M., Applied Surface Science, 371, pp. 322-328 (2016).

14. Zheng, S. F., Eimann, F., Philipp, C., Fieback, T., and Gross, U., Int. J. Heat Mass Transf., 120, pp. 879-894 (2018).

15. Anand, S., Condensational Droplet Growth in Rarefied Quiescent Vapor and Forced Convective 
Conditions, Dissertations \& Theses - Gradworks (2011).

16. Seo, D., Oh, S., Shin, S., and Nam, Y., Int. J. Heat Mass Transf., 114, pp. 934-943 (2017).

17. Wu, X.M.,Chu, F. and Chen, Y., Journal of Chemical Industry and Engineering, 66(s1), pp. 6064 (2015). 